\title{
Detecting transitions in protein dynamics using a recurrence quantification analysis based bootstrap method
}

\author{
Wael I. Karain
}

\begin{abstract}
Background: Proteins undergo conformational transitions over different time scales. These transitions are closely intertwined with the protein's function. Numerous standard techniques such as principal component analysis are used to detect these transitions in molecular dynamics simulations. In this work, we add a new method that has the ability to detect transitions in dynamics based on the recurrences in the dynamical system. It combines bootstrapping and recurrence quantification analysis. We start from the assumption that a protein has a "baseline" recurrence structure over a given period of time. Any statistically significant deviation from this recurrence structure, as inferred from complexity measures provided by recurrence quantification analysis, is considered a transition in the dynamics of the protein.

Results: We apply this technique to a 132 ns long molecular dynamics simulation of the $\beta$-Lactamase Inhibitory Protein BLIP. We are able to detect conformational transitions in the nanosecond range in the recurrence dynamics of the BLIP protein during the simulation. The results compare favorably to those extracted using the principal component analysis technique.

Conclusions: The recurrence quantification analysis based bootstrap technique is able to detect transitions between different dynamics states for a protein over different time scales. It is not limited to linear dynamics regimes, and can be generalized to any time scale. It also has the potential to be used to cluster frames in molecular dynamics trajectories according to the nature of their recurrence dynamics. One shortcoming for this method is the need to have large enough time windows to insure good statistical quality for the recurrence complexity measures needed to detect the transitions.
\end{abstract}

Keywords: Recurrence quantification analysis- principal component analysis - molecular dynamics

\section{Background}

Protein functional motions occur over a wide range of time scales, and are usually accompanied by conformational transitions in the protein [1]. Principal component analysis PCA is a standard technique used to detect conformational transitions based on molecular dynamics MD simulations [2-9]. This is done by first removing the overall rotations and translations for the protein atoms by aligning each frame in the simulation to a reference frame. The covariance matrix for a set of atoms, usually the $C_{\alpha}$ atoms, is then built up. It is given by

Correspondence: wqaran@birzeit.edu

Department of Physics, Birzeit University, P.O.Box 14, Birzeit, Palestine

$$
C_{i j}=<\left(X_{i}-X_{i, a}\right)\left(X_{j}-X_{j, a}\right)>
$$

where $X$ are the $x, y, z$ coordinates for the $C_{\alpha}$ atoms fluctuating about their average positions $X_{a}$. Collective motion coordinates are prepared by diagonalizing this covariance matrix. This provides a set of eigenvalues and their corresponding eigenvectors. Each eigenvector corresponds to a collective motion direction in $3 \mathrm{~N}$ space, where $\mathrm{N}$ is the number of protein residues. The corresponding eigenvalue represents the total mean square fluctuation of all the residues in that direction. The projection of motion for the protein is then calculated along any given eigenvector to show any conformational transitions over time. A small set of eigenvectors is usually sufficient to provide the majority of the fluctuations. In its standard form, this technique detects only linear 
correlations. Even though it has been extended to detect nonlinear correlations, its ability in this field is still not very satisfactory $[2,6]$, and while it is not limited to harmonic motions, some nonlinear relationships might be misinterpreted due to the neglecting of higher order correlations [6]. In addition, PCA depends on the time window length used to compute the eigenvalues and eigenvectors [10]. Conformational transitions can also be tracked by calculating the root mean square deviation RMSD for a set of atoms, such as the backbone $C_{\alpha}$ atoms, from a reference structure. Large changes in the RMSD usually point to conformational transitions. However, intermediate values of RMSD are sometimes hard to interpret [11].

In this work, we propose a different approach to detecting conformational transitions. We start by assuming that during any given time period, a protein has a 'baseline' recurrence structure. When it is undergoing a conformational transition, it will deviate from this baseline recurrence state. If this significant deviation is detected, then one can point to a conformational transition taking place. To achieve this goal, we will use recurrence quantification analysis RQA, which has gained popularity in studying dynamics, transitions, and synchronization in dynamical systems [12-14]. RQA is a quantitative version [15] of recurrence plots RP, which visually highlight recurrences in dynamical systems [12]. It is used in many fields [14]. In particular, RPs and RQA have been used extensively to study proteins over the years [16-54].

The RP is a non-linear analysis method. It visualizes graphically the recurrence $S_{i}$ to a former state $S_{j}$ in the phase space trajectory of the dynamical system. It is most useful when the system is being investigated experimentally, with an unknown theoretical time evolution law. If a scalar time-series $\left\{U_{i}\right\}$ is available for one of the measurable quantities for this system, then its trajectory can be reconstructed [55]. This reconstruction involves using the method of time delays. In essence, the dynamics of the system are assumed to be encapsulated in the time-series for the single measurable quantity, with the time delays approximating derivatives [56]. The m-dimensional phase space orbit is re-constructed from the scalar time series $U_{i}$, such that.

$$
S_{i}=\left(U_{i}, U_{i+d}, \ldots . . U_{i+(k-l) d}\right) k=l, . . m
$$

where $d$ is the delay parameter between the timedelayed versions, and mis the embedding dimension for the reconstructed phase space. The embedding dimension $m$ represents the degrees of freedom (or the number of dominant operating variables) in the dynamical system of interest. It is estimated by the method of false nearest neighbors [57]. The delay parameter $d$ determines the number of points to be skipped in the scalar time-series $U_{i}$ between the numbers forming the m-dimensional vector $S$. It is set to a value that makes the correlation between the points of the measured timeseries at a minimum, and is estimated by finding the first minimum in the mutual information function [58]. This m-dimensional vector in phase space, $S_{i}$, represents the state of the system at time $i$. The RP is prepared by assigning a dot at each point $(i, j)$ whenever a point $S_{j}$ lies within a ball of radius $\varepsilon$ centered at $S_{i}$. In other words, if two vectors representing the state of the system are within a certain tolerance from each other, then this means that the system is in similar states at two different time instances, $i$ and $j$. The mathematical expression of the RP matrix is:

$$
R_{i, j}(\varepsilon)=\Theta\left(\varepsilon-\left\|S_{i}-S_{i}\right\|\right) \quad i, j=1, \ldots \ldots \ldots ., N
$$

where $N$ is the number of states, $\varepsilon$ is a threshold distance, $\Theta$ is the Heaviside function $(\Theta(x)=0$ if $x<0$ and 1 otherwise), and $\|\cdot\|$ is chosen from one of the frequently used norms: the $\mathrm{L}_{1}$-norm(Minimum norm), the $\mathrm{L}_{2}$ norm (Euclidean norm), and the $\mathrm{L}_{\infty}$ (Maximum norm). The norm parameter determines the size and shape of the neighborhood surrounding each reference point. In this work the maximum norm is used. The ratio of the number of dots to the total number of points in the matrix gives the recurrence rate value $R R$. The threshold or radius parameter $\varepsilon$ is the limit that transforms the distance matrix (DM) between the time points into a recurrence matrix (RM). It plays a role similar to that of the Heaviside function. Elements $(i, j)$ in the DM with distances between states at or below the radius cutoff are included in the RM $\left(R_{i, j}=1\right)$. Elements above the cutoff are excluded from RM $\left(R_{i, j}=0\right)$. This threshold can be chosen using a number of different techniques. For example, one rule of thumb is to choose a threshold that gives a RR value of $1 \%$ [14]. However, the value of $\varepsilon$ is usually chosen according to the application at hand [14].

In addition to $R R$, RQA provides other output parameters. We will concentrate on two of these: determinism, DET, and laminarity, LAM. DET is the fraction of recurrence points forming diagonal lines parallel to the central diagonal. It is given by

$$
D E T=\sum_{l=l \min }^{N} l P(l) / \sum_{l=1}^{N} l P(l)
$$

where $l_{\min }$ defines the minimal length for a diagonal line and is usually taken to be 2 [14]. $P(l)$ is the probability distribution for the diagonal line lengths. The length of diagonal lines depends on the dynamics of the system [14]. A large number of long diagonal lines points to a 
high predictability of the system, and to the fact that it evolves at a similar fashion at different points in time. While the value of DET might point to a deterministic nature of a system, this is not a sufficient condition [59].

LAM is the fraction of recurrence points forming vertical structures, and is given by

$$
L A M=\sum_{l=l \min }^{N} v P(v) / \sum_{l=1}^{N} v P(v)
$$

where $l_{\text {min }}$ defines the minimal length for a vertical line and is usually taken to be 2 [14]. $P(v)$ is the probability distribution for the vertical line lengths. Vertical structures in the recurrence plot point to slowly changing states, common during laminar phases [14].

Changes in complexity measures provided by RQA, such as DET and LAM, are generally interpreted as pointing to transitions in the dynamics. In some cases, the relative values of $R R$ and DET are used to detect transitions in the dynamics [60]. However, this has usually been done without providing confidence intervals to validate the significance of these changes. Recently, a method based on bootstrapping has been proposed to remedy this deficiency [61, 62]. It easily provides confidence intervals for analysis within a single dynamical system.

The method starts by preparing the recurrence matrix over a moving time window of length $w$, with the starting point of the time window at the beginning of the time series. This starting point is then shifted by a suitable number of time steps forward, and the process repeated, until the end of the time series is reached. For each time window, the local distribution for the diagonal line lengths is prepared. The distributions from all the windows are then merged together to prepare one global distribution of diagonal line lengths. This distribution is consequently used to calculate the global complexity measure of interest for the system, which will be DET in our case. From this global distribution, a large number of bootstrap distributions are drawn, and the value for DET is calculated for each draw. The $\alpha$ quantiles are subsequently calculated, and their corresponding confidence levels prepared for the DET distribution [62]. It is assumed that DET values above the high confidence level, and those below the low confidence level, point to a significant change in the dynamics of the system from its assumed baseline recurrence dynamics state. A similar procedure is applied to the vertical line distributions to prepare confidence levels for LAM [62].

In this work we will apply this RQA based bootstrap approach to detect changes in dynamics over a 132 ns long molecular dynamics simulation, for the 165 residue $\beta$-Lactamase Inhibitory Protein BLIP at $310 \mathrm{~K}$. This protein is secreted by the soil bacterium Streptomyces clavuligerus. It inhibits $\beta$-lactam enzymes, which hydrolyze $\beta$-lactam antibiotics and nullify their effect [63-65]. It consists of five alphahelices, and eight beta-sheets. It also has distinct connecting loops (Fig. 1).

\section{Methods and calculations}

The computer programs VMD [66], and NAMD [67], are used to perform the molecular dynamics simulation, and the associated analyses respectively. The CHARMM27 par_all27_prot_lipid.inp parameter file is used for the force field. The starting BLIP protein structure is downloaded from the protein data bank (PDB entry 3gmu) [68]. Periodic boundary conditions are used in an $80 \AA x$ $80 \AA \times 80 \AA$ box. The protein is neutralized using $20 \mathrm{Cl}^{-}$ ions and $22 \mathrm{Na}^{+}$ions. The protein is solvated using 15,264 TIP3P waters $(0.15 \mathrm{M} / \mathrm{NaCl})$. The Particle-Mesh-Ewald method is used to do the electrostatic calculations [69]. A switching function is used for non-bonded interactions with a switch distance of $10 \AA$ and a cutoff distance of $12 \AA$. A pair-list distance of $14 \AA$ is used. The simulation is performed at constant pressure of 1 atm with an integration step of $2 \mathrm{fs}$. Langevin dynamics are used to control both temperature and pressure. A langevin temperature damping coefficient of 10/ps is applied. A langevin piston period of $200 \mathrm{fs}$ and a langevin piston decay period of $100 \mathrm{fs}$, are used respectively. The protein is minimized using the conjugate gradient method for 5000 steps $(10 \mathrm{ps})$ to relax any high energy areas in the system. This is followed by a gradual heating protocol in small temperature steps of $10 \mathrm{~K}$ to avoid thermal instability. Starting from an initial temperature of $100 \mathrm{~K}$, langevin dynamics is used to increase the temperature by $10 \mathrm{~K}$

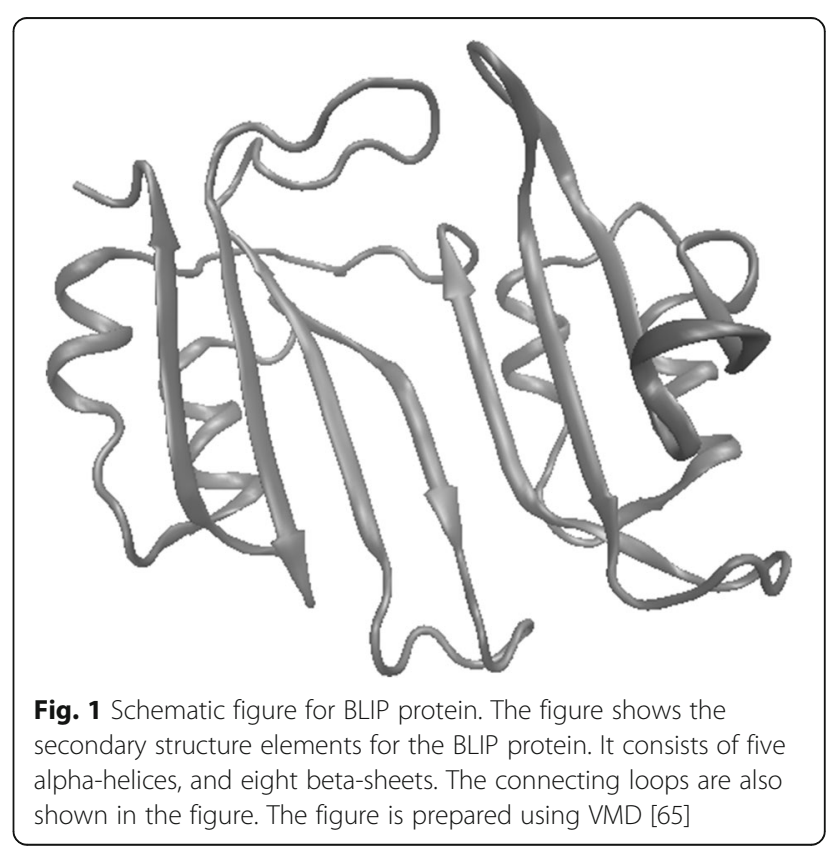




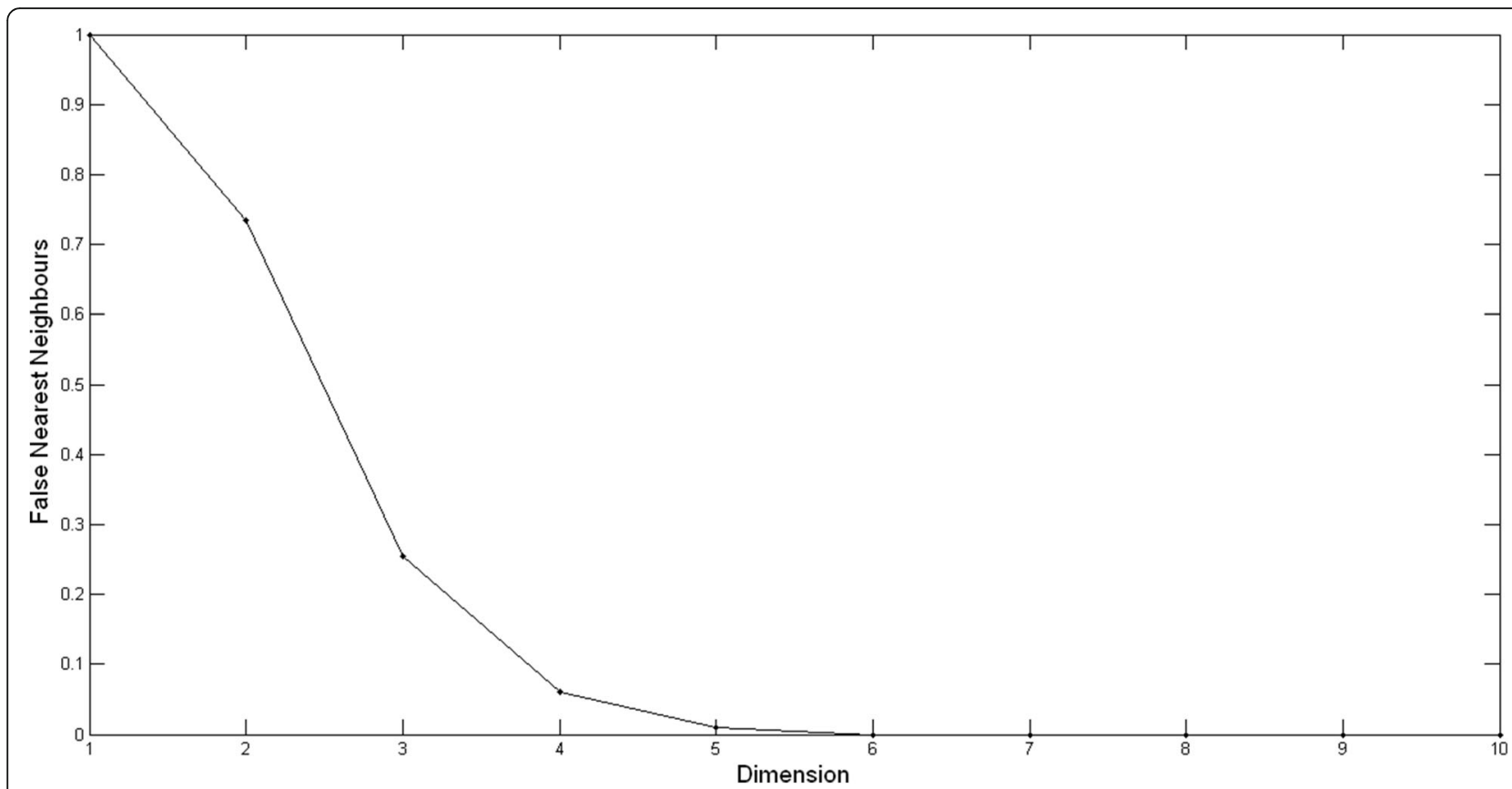

Fig. 2 The embedding dimension. The embedding dimension value of 6 is calculated using the false nearest neighbor method in the cross recurrence CRP toolbox [69]
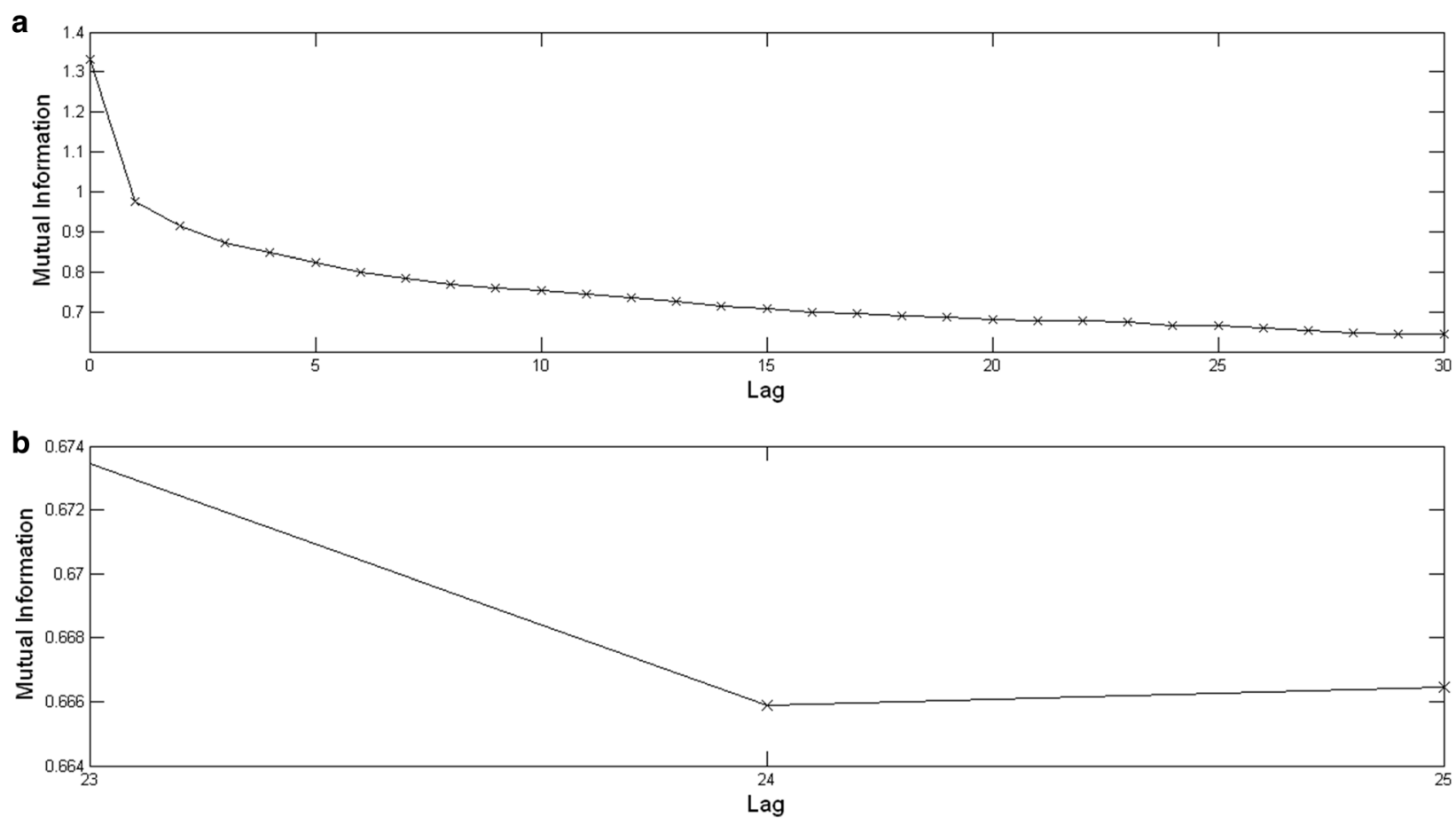

Fig. 3 The delay parameter. The delay parameter value of 24 is calculated using the mutual information method in the cross recurrence CRP toolbox [69]. a This top graph shows the mutual information values for the lag range 0 to 30. $\mathbf{b}$ This bottom graph shows a magnified version of the top graph in (a), exhibiting the first mutual information minimum at a lag of 24 . This value is used for the delay parameter 


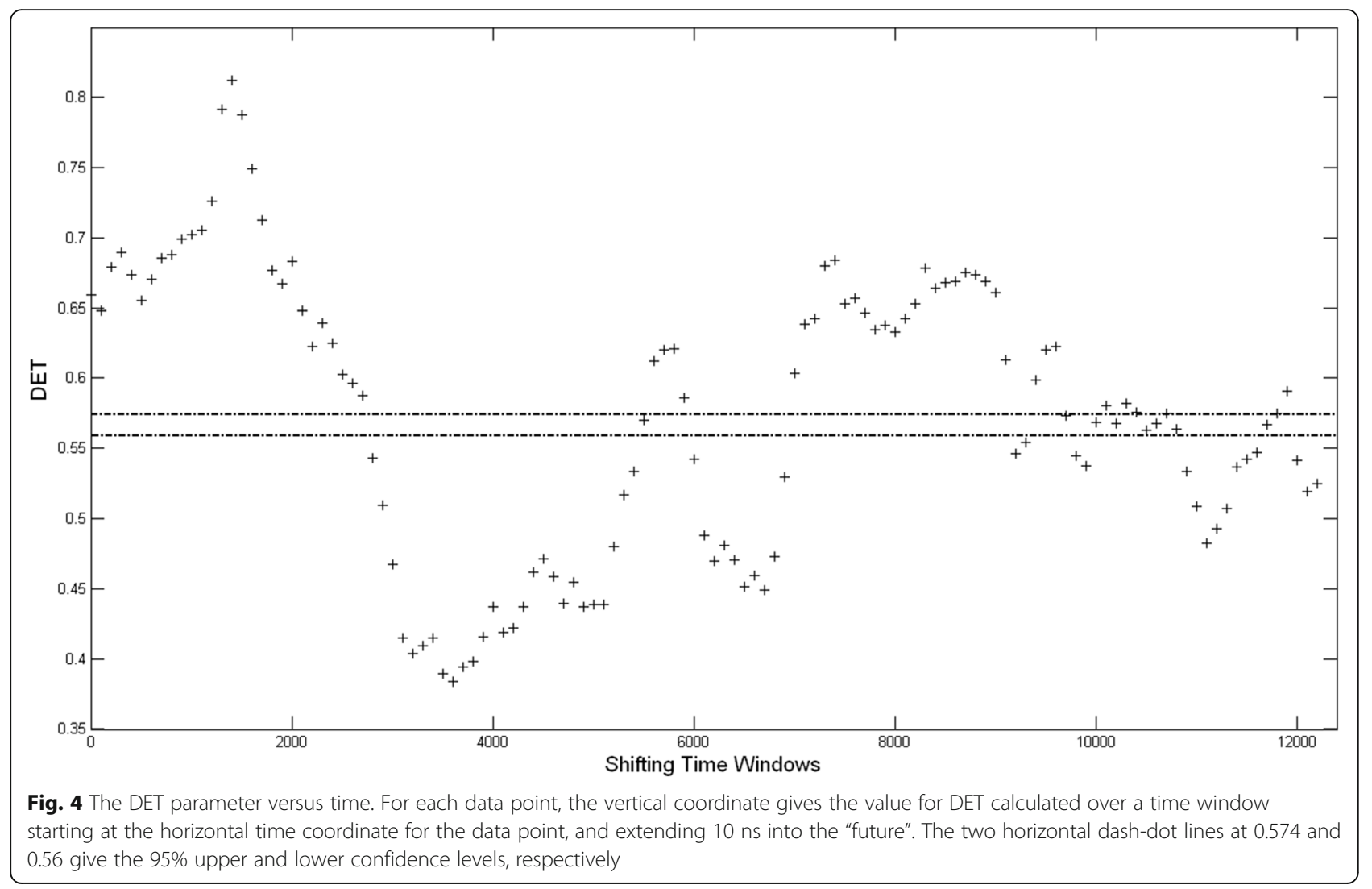

steps, and to control the temperature using a damping coefficient of $10 / \mathrm{ps}$. The simulation is run for $10 \mathrm{ps}$ for each $10 \mathrm{~K}$ temperature step. This is continued until reaching the final simulation temperature of $310 \mathrm{~K}$. The equilibration period is $5 \mathrm{~ns}$ long. To insure equilibration, a number of parameters (potential energy, kinetic energy, temperature, pressure, RMSD) are tested for convergence. A 132 ns production run is consequently prepared with an integration time step of $2 \mathrm{fs}$. A root mean square deviation RMSD series is prepared using VMD for the carbon alpha atoms in the protein. The time series is 13,200 points long, with a time spacing of 10 ps between time points, for a total time length of $132 \mathrm{~ns}$. The parameters for the phase space trajectory reconstruction are prepared using the CRP toolbox subroutines [70]. The maximum norm is used. The embedding dimension is prepared using the false nearest neighbor FNN subroutine and has a value of 6 (Fig. 2).

The delay parameter is prepared using the mutual information MI subroutine, and has a value of 24(Fig. 3).

The epsilon value for each time window is adaptively chosen to give a constant RR of $5 \%$. The data inside each window is normalized. The DET and LAM parameters are subsequently calculated for a time window that is 1000 points long (10 ns) using the CRQA subroutine in the CRP toolbox [70]. The time window is then shifted by $1 \mathrm{~ns}$, and the DET/ LAM calculation repeated using the same procedure above until we reach the end of the time series. The diagonal line lengths and the vertical line lengths, from all the time windows, are then binned into their corresponding one global distribution. Each global distribution is then bootstrapped 1000 times. For each bootstrap copy, the number of recurrence structures drawn is the mean number of recurrence structures contained in the local distributions, and subsequently, DET and LAM are calculated [62]. Once the DET and LAM distributions are prepared, the 95\% quantiles are calculated, and the corresponding upper and lower confidence levels are derived for both DET and LAM respectively. The PCA analysis is performed using the CARMA program [71].

\section{Results and discussion}

Figure 4 shows the results for the DET recurrence parameter versus time over the 132 ns simulation. The data points are spaced 1 ns apart. Each data point gives the value of DET for a time window that begins at that time instant, and extends $10 \mathrm{~ns}$ into 


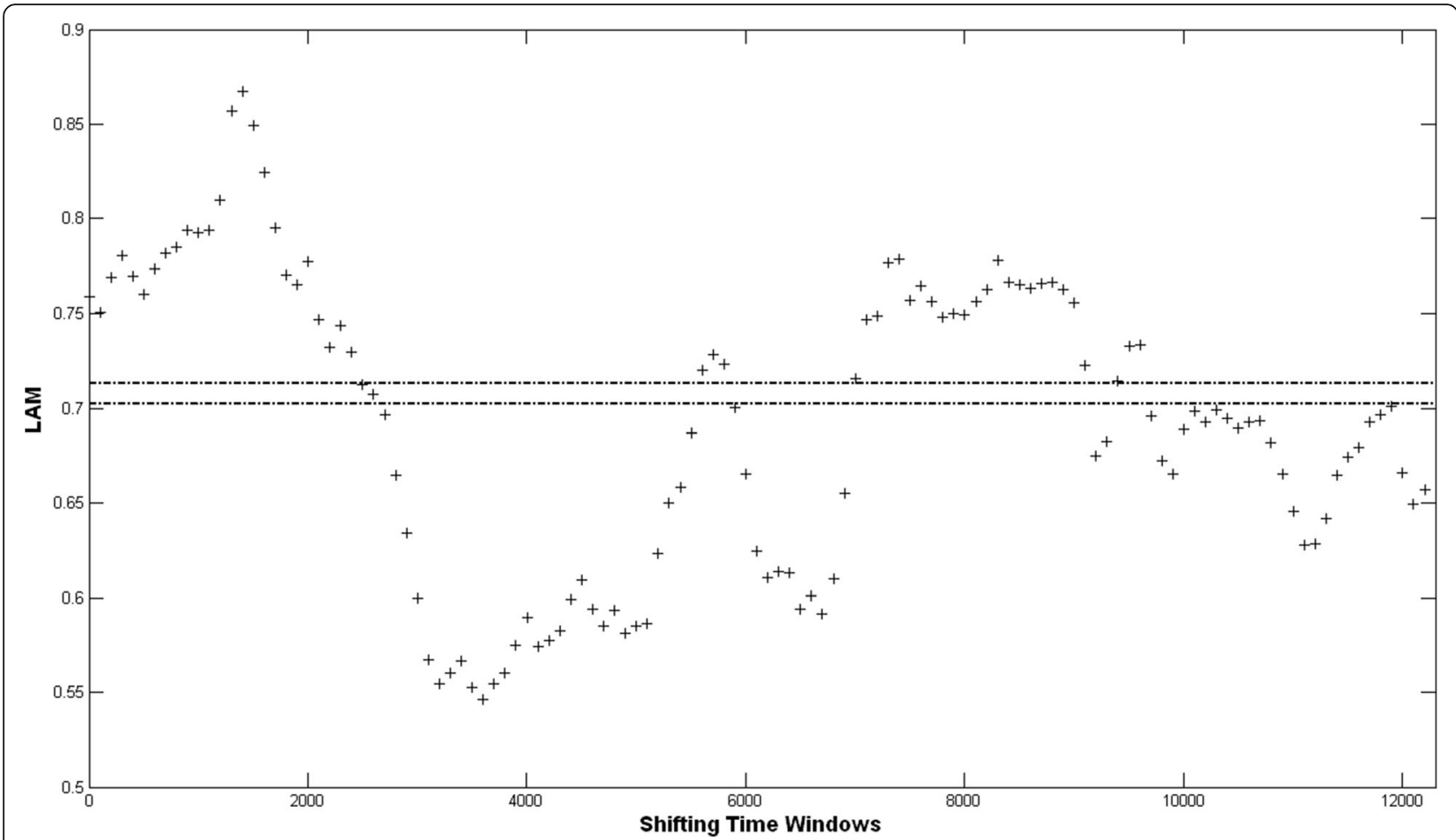

Fig. 5 The LAM parameter versus time. For each data point, the vertical coordinate gives the value for LAM calculated over a time window starting at the horizontal time coordinate for the data point, and extending $10 \mathrm{~ns}$ into the "future". The two horizontal dash-dot lines at 0.713 and 0.702 give the $95 \%$ upper and lower confidence levels, respectively

the "future". For example, the value for DET at 10 ns represents the DET value for the time window starting at $10 \mathrm{~ns}$ and ending at $20 \mathrm{~ns}$. The starting point for each window is shifted forward by 1 ns relative to the previous window. Thus the DET value at $11 \mathrm{~ns}$ is for the window starting at $11 \mathrm{~ns}$ and extending to 21 ns. The two dash-dot horizontal lines at 0.574 and 0.56 show the upper and lower 95\% confidence levels respectively. The time points where the value of DET is above 0.574 or below 0.56 , delineate regions with significantly different recurrence dynamics than the assumed baseline recurrence dynamic state. In other words, a transition in dynamics occurs where the DET value crosses these two horizontal dash-dot lines. Both upper and lower confidence levels are given since the exact nature of the dynamics is not known [62]. Inspection of Fig. 4 shows three time regions with a DET value larger than the upper confidence level: 0 ns-27 ns, $55 \mathrm{~ns}-60 \mathrm{~ns}$, and $72 \mathrm{~ns}-$ $91 \mathrm{~ns}$. In addition, there are three regions with DET values below the lower confidence level: $28 \mathrm{~ns}-55 \mathrm{~ns}$, 60 ns-69 ns, and 108 ns-115 ns. Again, one needs to emphasize here that each time point in these six regions actually denotes a time window starting at that point, and extending $10 \mathrm{~ns}$ into the 'future'. We recalculate DET with a constant $R R$ of $1 \%$ within each time window. This is done to insure that the results are independent of the relatively high $R R 5 \%$ value chosen for this application. The calculated DET values at $1 \%$ are shifted downwards relative to those at 5\%. The 95\% confidence levels are also shifted downwards. However, the time regions above and below the corresponding confidence intervals are essentially the same for both $5 \%$ and $1 \%$. Thus the choice of $5 \%$ is justified since it has the added advantage of improving the statistical reliability of the calculations by increasing the number of recurrence structures in each time window. We also repeat the bootstrap analysis with a constant recurrence threshold value in each time window, instead of a constant $R R$ value. This results in large fluctuations in the number of recurrence structures -diagonal and vertical lines- within each time window, and strongly limits the use of this bootstrap technique, which depends on having a constant statistical sample within each time window.

In these six regions, the dynamics of the protein is significantly different than the "baseline" state which is assumed to fall between the two confidence level lines. The regions with a DET value larger than the 
upper limit hint at an increased regularity and autocorrelation in the system, while those below the lower limit point to more irregularity and stochastic variability in the system dynamics [62]. One needs to point out that while this method detects transitions in dynamics, it does not provide a clear picture for the nature of the dynamics; only that the system has deviated from its baseline recurrence state. Another point to keep in mind is that the exact time point where the transition takes place is not well defined because each DET value is calculated over long overlapping time windows.

Figure 5 shows the results from the analysis on LAM. It is clear that both DET and LAM give very similar outcomes in terms of the time windows with LAM values above and below the $95 \%$ confidence levels respectively. We will therefore only use the results we get from DET for the rest of the paper.

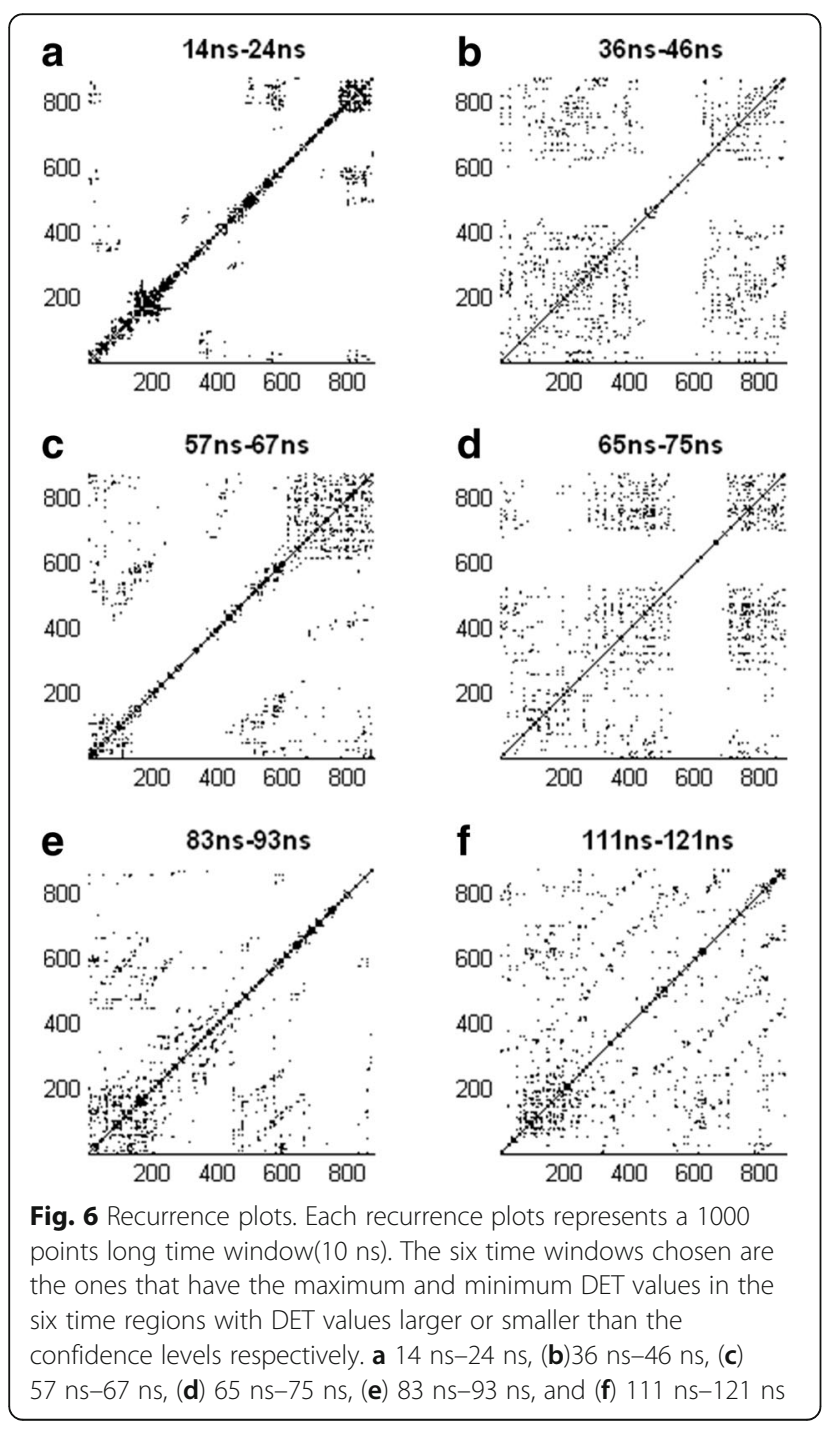

Representative recurrence plots from the six regions are shown in Fig. 6. Each recurrence plot represents a time window 1000 points long $(10 \mathrm{~ns})$. The plots in (a), (c), and (e) are each extracted from one of the three regions with DET values above the 95\% confidence level, and have the maximum DET values within their corresponding regions. The plots in (b), (d), and (f) are each extracted from one of the regions with DET values below the 95\% confidence level, and have the minimum DET values within their corresponding regions. While it is difficult to draw clear and objective conclusions based only on visual inspection of these recurrence plots, it can be seen that the plots from the three regions with large DET values have a large proportion of their recurrence structures near the main diagonal. On the other hand, the plots with the small DET values are spread out over the entire plot.

To gain a better picture of where these six regions lie in the conformational space, we resort to PCA. We limit our analysis to the first three principal components PCs, which constitute $46 \%$ of the total fluctuations in our simulation(Fig. 7).

Figure 8 gives the two dimensional projection of the 132 ns simulation on principal component $1 \mathrm{PC} 1$ and principal comonent $2 \mathrm{PC} 2$, as the horizontal axis and vertical axis respectively. K-means clustering and the 'elbow' technique [72] are used to cluster the data. Four distinct regions emerge: cluster I, II, III, and IV, respectively.

In Fig. 9 we project the same six 10 ns time windows shown as recurrence plots in Fig. 5, along PC1 and PC2(projections in black color). In (a), the $14 \mathrm{~ns}-24 \mathrm{~ns}$ window falls inside the red cluster III. In (b), the $36 \mathrm{~ns}-46 \mathrm{~ns}$ window lies mainly inside the blue cluster I. In (c), the $57 \mathrm{~ns}-67 \mathrm{~ns}$ window straddles the blue cluster I and the green cluster II, and dips slightly into the violet cluster IV. In (d), the 65 ns-75 ns window falls mainly inside the green cluster II. In (e), the 83 ns-93 ns window also falls mainly within the green cluster II. Finally in (f), the 111 ns-121 ns window is situated inside the violet cluster IV. We notice that with the exception of the $57 \mathrm{~ns}-67 \mathrm{~ns}$ window, the windows fall mainly within a single cluster each. It is also interesting to note that time windows with large and small DET values fall within the same cluster(Fig. 9d and e). This shows that while PCA lumps regions with distinct dynamics within the same cluster, while the RQA-bootstrap method is able to resolve them apart.

Figure 10 gives the two dimensional projection of the 132 ns simulation on principal component $1 \mathrm{PC} 1$ and principal comonent $3 \mathrm{PC} 3$, as the horizontal axis and vertical axis respectively. K-means clustering and the 


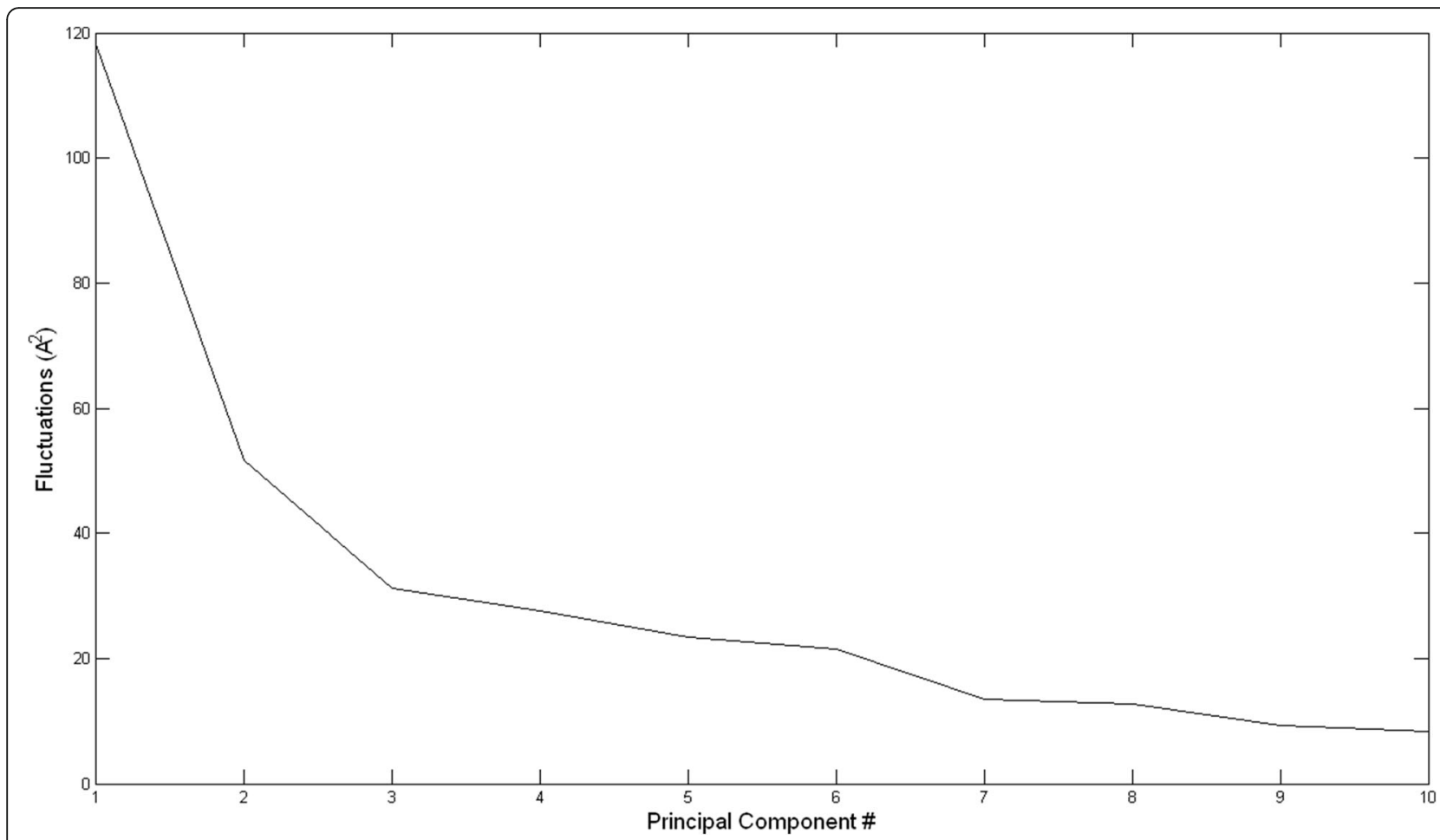

Fig. 7 Positional fluctuations for principal component modes. The first three principal components constitute $46 \%$ of the total positional fluctuations

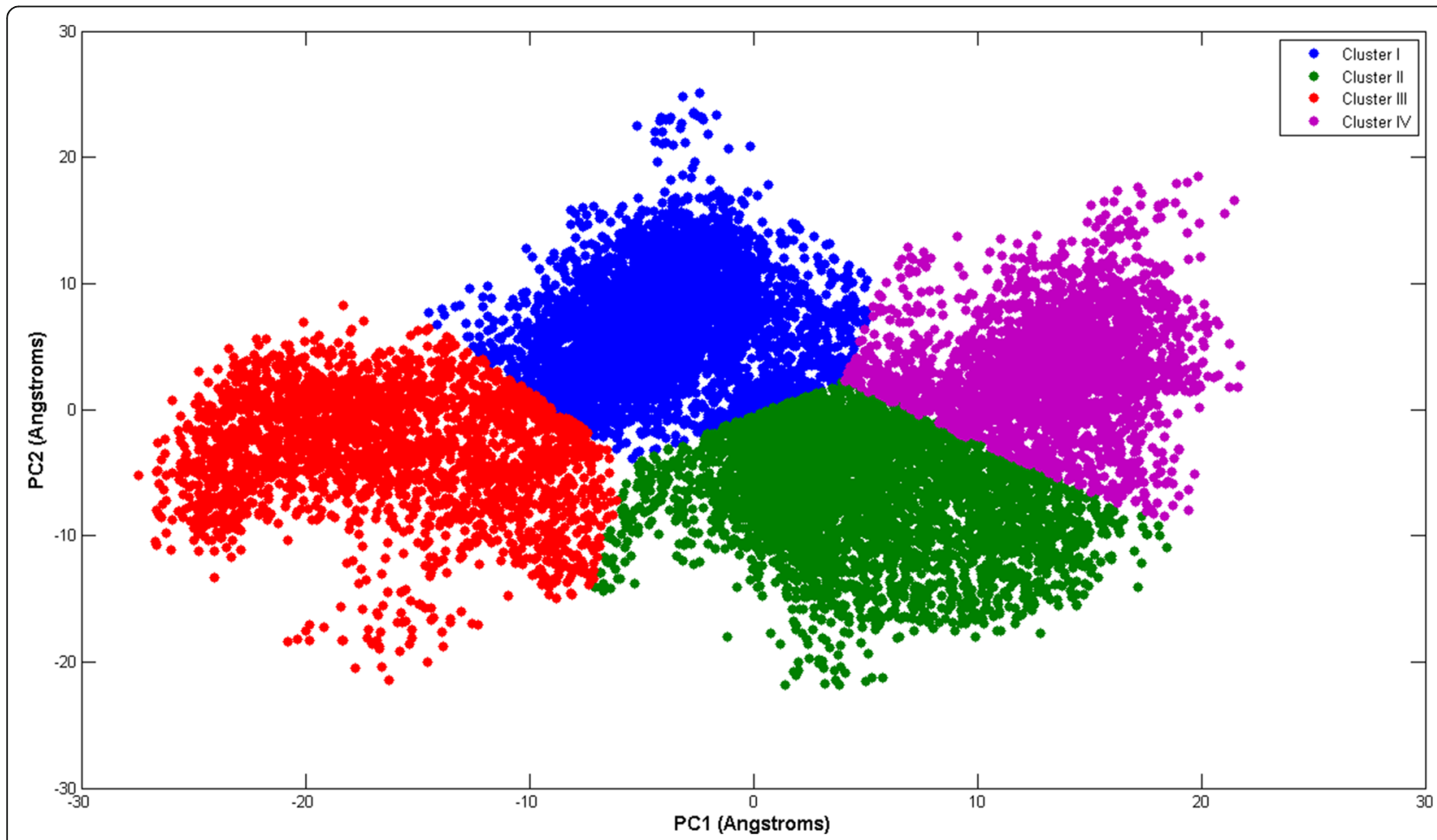

Fig. 8 gives the two dimensional projection of the 132 ns simulation on principal component 1 PC1 and principal comonent 2 PC2, as the horizontal axis and vertical axis respectively. K-means clustering and the 'elbow' technique [72] are used to cluster the data. Four distinct regions emerge: cluster I, II, III, and IV, respectively 

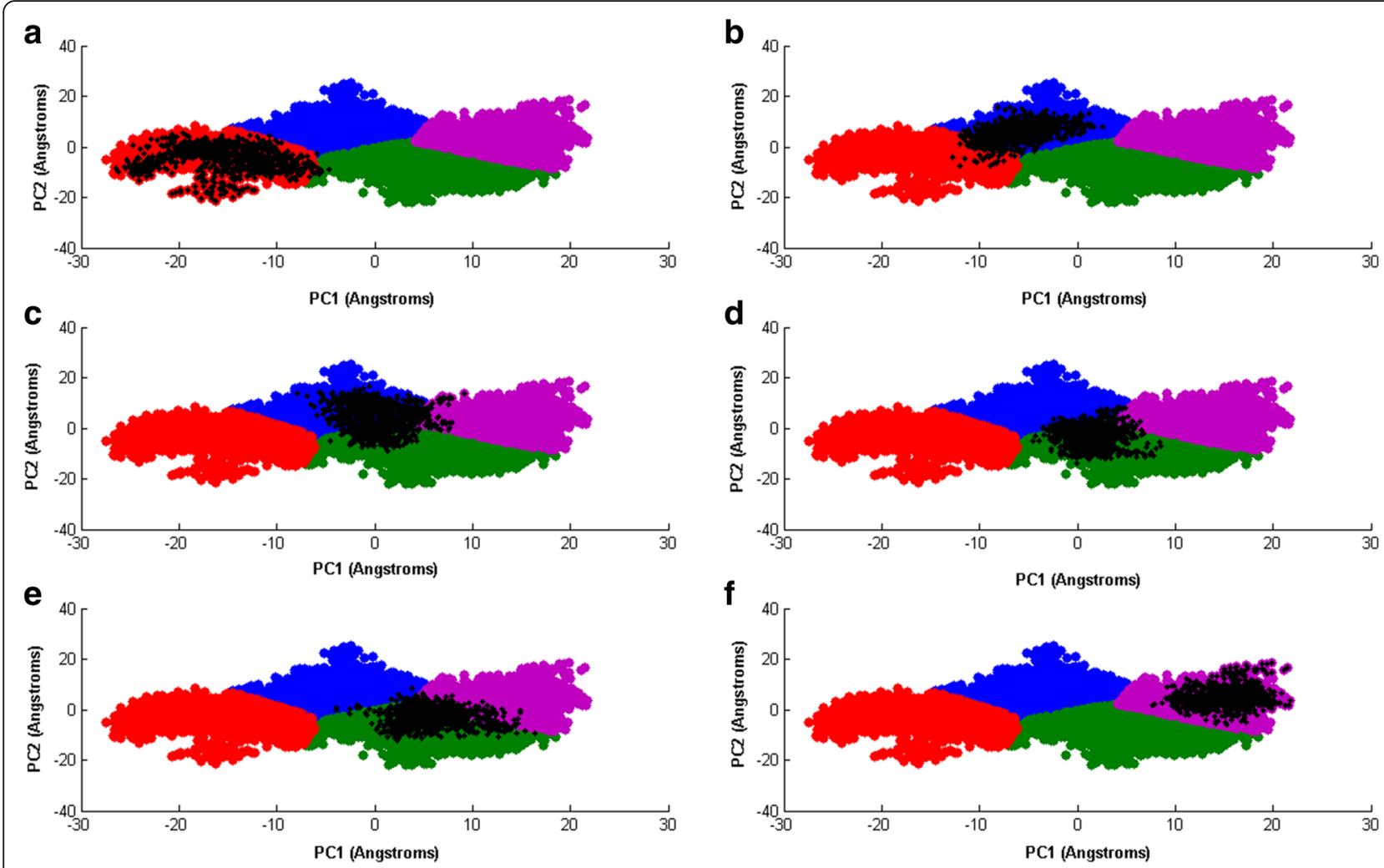

Fig. 9 The projection of time windows over a plane defined by PC1 and PC2. The time window projections are in black: (a) 14 ns-24 ns(large DET), (b) 36 ns-46 ns(small DET), (c) 57 ns-67 ns(large DET), (d) 65 ns-75 ns(small DET), (e) 83 ns-93 ns(large DET), (f) 111 ns-121 ns(small DET)

'elbow' technique [72] are used to cluster the data points. Five distinct regions emerge: cluster I, II, III, IV, and $\mathrm{V}$, respectively.

In Fig. 11 we project the same six 10 ns time windows shown as recurrence plots in Fig. 6, along PC1 and PC3(projections in black color). In (a), the $14 \mathrm{~ns}-24 \mathrm{~ns}$ time window falls mainly inside the blue cluster III. In (b), the 36 ns-46 ns time window lies mainly inside the red cluster I. In (c), the 57 ns-67 ns time window is inside the yellow cluster V. In (d), the 65 ns-75 ns time window also falls inside the yellow cluster V. In (e), the 83 ns-93 ns time window also falls mainly within the green cluster IV. Finally in (f), the 111 ns-121 ns window is situated inside the brown cluster II. Again, we notice that the $57 \mathrm{~ns}-67 \mathrm{~ns}($ large DET) and $65 \mathrm{~ns}-$ $75 \mathrm{~ns}(\mathrm{small} \mathrm{DET})$ time windows fall within the yellow cluster $\mathrm{V}$, while the other four time windows fall mainly within a single cluster each. This again shows that while

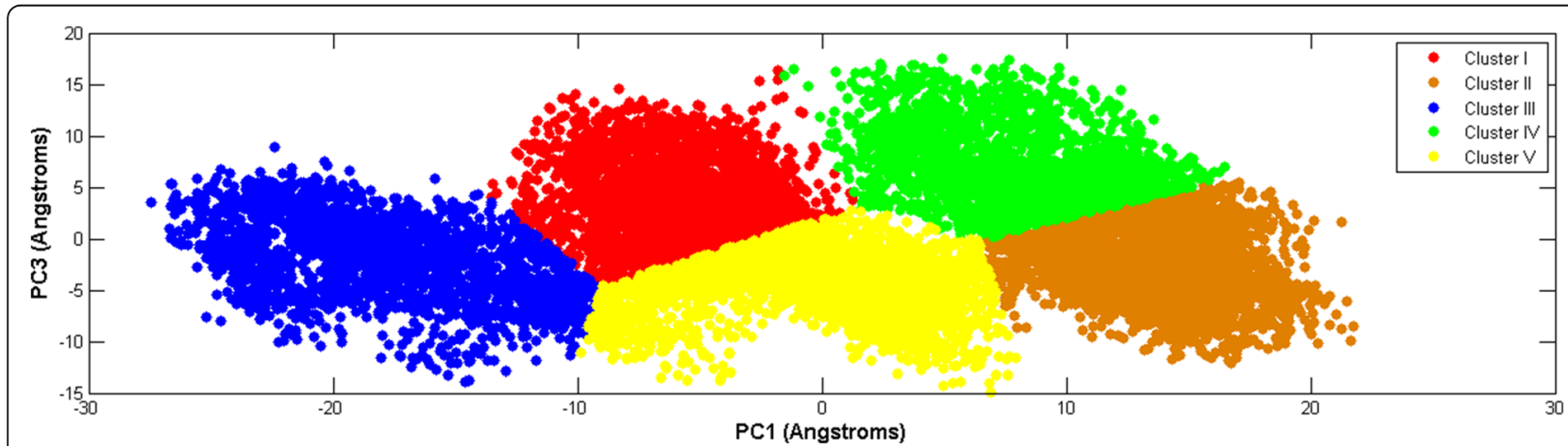

Fig. 10 The projection of the 132 ns simulation over PC1 and PC3. The five clusters I, II, III, IV, and V are grouped using k-means clustering and the 'elbow' technique 

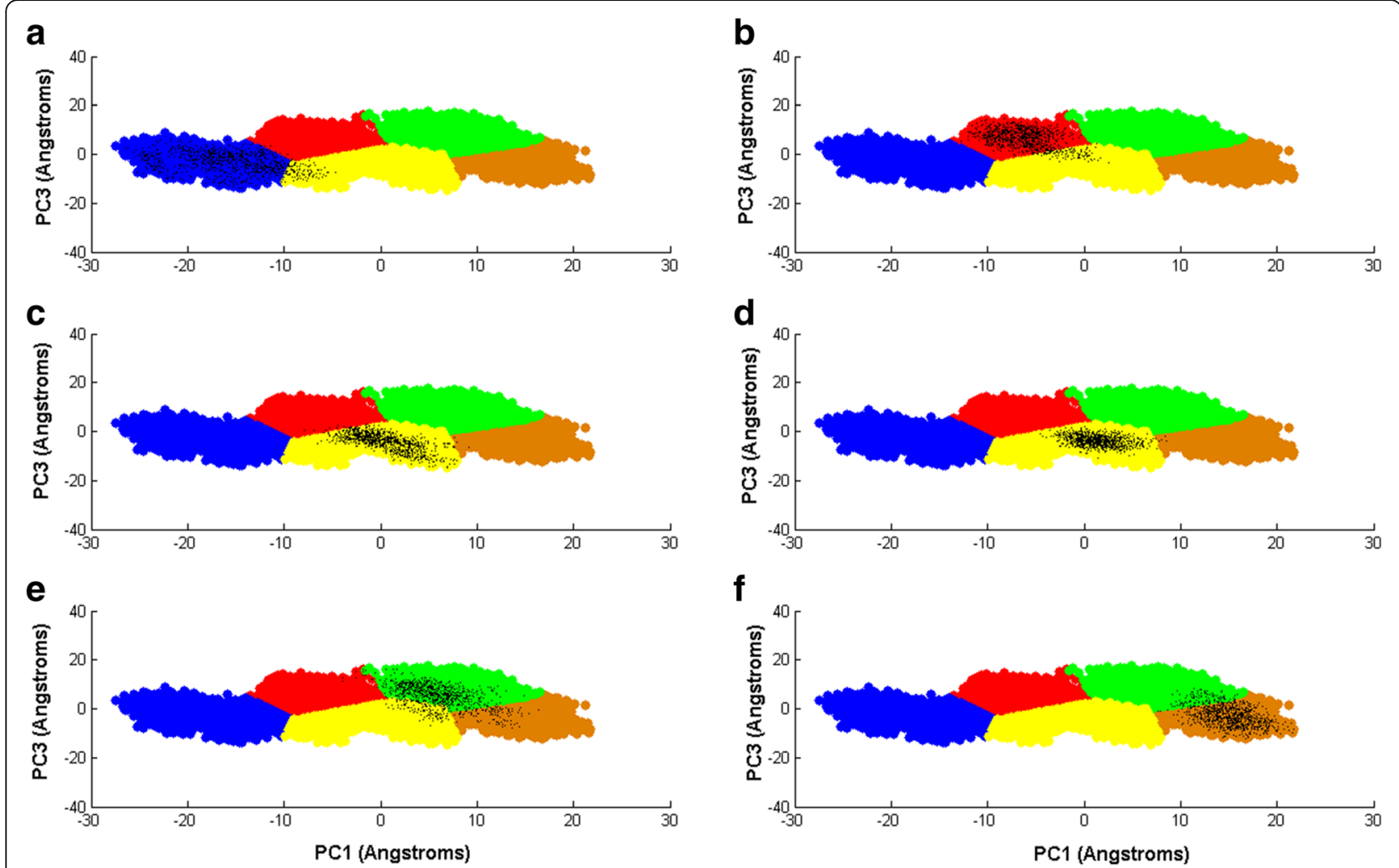

Fig. 11 The projection of time windows over a plane defined by PC1 and PC3. The time window projections are in black: (a)14 ns-24 ns(large DET), (b) 36 ns-46 ns(small DET), (c) 57 ns-67 ns(large DET), (d) 65 ns-75 ns(small DET), (e) 83 ns-93 ns(large DET), (f) 111 ns-121 ns(small DET)

PCA lumps regions with distinct dynamics within the same cluster, the RQA-bootstrap method is able to resolve them apart.

To gain some insight into the conformational structural nature of these transitions, we project two $10 \mathrm{~ns}$ long time windows over the principal components with the three largest eigenvalues, and show where the collective domain motion amplitudes are the largest. The first time window is from $14 \mathrm{~ns}$ to 24 ns (Fig. 12), and has a DET value larger than the upper 95\% confidence level. The second time window is from 36 ns to 46 ns (Fig. 13), and has a DET value smaller than the lower 95\% confidence level. It is clear that for both of these time windows, the collective domain motions are taking place mainly within the loop regions between the secondary structures of the protein. In Figs 12 and 13, there are five regions with clear collective loop domain motions. In region a, the loop lies between beta-sheets 6 and 7. In region $\mathbf{b}$, the loop lies between beta-sheets 2 and 3 . In region c, the loop lies between the short alpha-helix 3 and beta-sheet 2 . In region $\mathbf{d}$, the loop lies between beta-sheets 3 and 4 . In region e, the loop lies between beta-sheets 5 and 6 . Such loop structural conformations can play an important role in protein docking and active site stabilization [73-80]. For BLIP in particular, residue Asp-49 which lies within region $\mathbf{b}$ in the loop between beta-sheets 2 and 3 , and residue Phe-142 which lies within region a in the loop between beta-sheets 6 and 7, play an important role in the inhibition behavior for the protein [81].

\section{Conclusions}

We have introduced a RQA based bootstrap method to differentiate between different recurrence dynamics regions in a protein molecular dynamics simulation. The nature of the dynamics is specifically related to the recurrence characteristics of the dynamical system. The method compares well with PCA. In addition, while PCA shows that certain time regions fall within a single cluster in conformational space, they actually have different recurrence qualities. This method can thus be used in unison with PCA to clarify the degree of correlation and predictability during a certain time window. It can also be used to cluster molecular dynamics trajectory data based on 


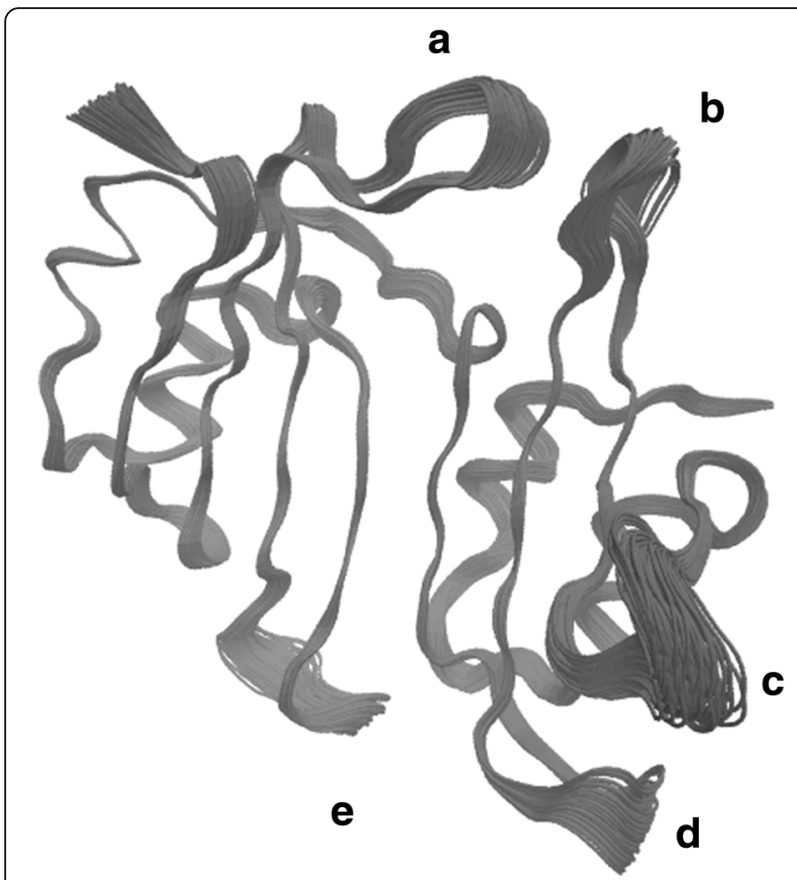

Fig. 12 Collective protein carbon alpha motions projected over PC1, PC2, and PC3 simultaneously. The projection of time window 14 ns24 ns, with DET value larger than the upper 95\% confidence level, simultaneously along the principal component eigenvectors with the three largest eigenvalues. Regions $\mathbf{a}, \mathbf{b}, \mathbf{c}, \mathbf{d}$, and e show clear collective loop domain motions. The figure is prepared using VMD [65]

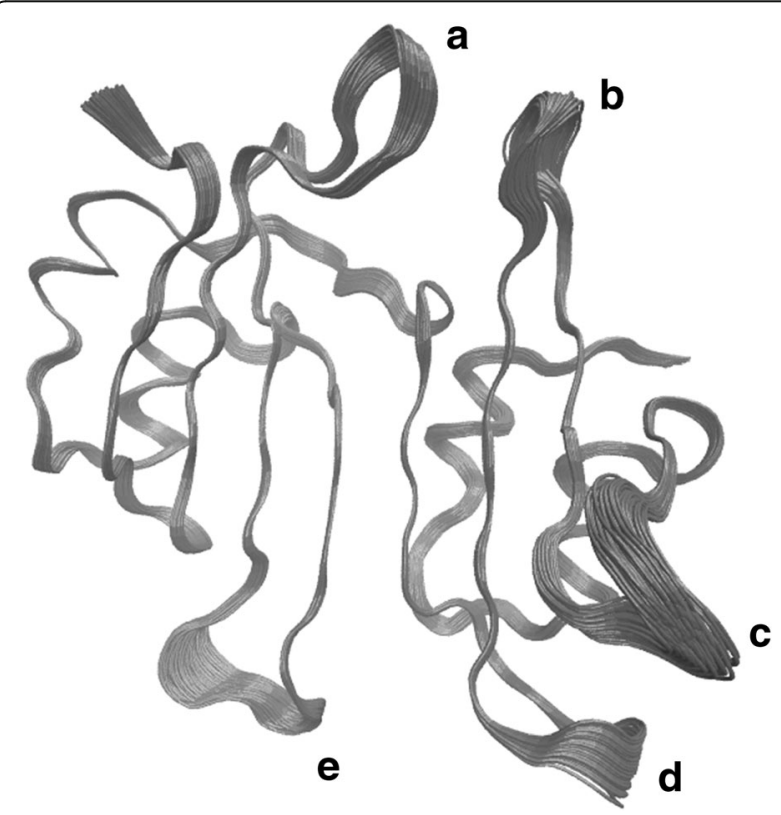

Fig. 13 Collective protein carbon alpha motions projected over PC1, PC2, and PC3 simultaneously. The projection of time window 36 ns46 ns with DET value smaller than the lower 95\% confidence level, simultaneously along the 3 PC eigenvectors with the three largest eigenvalues. Regions $\mathbf{a}, \mathbf{b}, \mathbf{c}, \mathbf{d}$, and e show clear collective loop domain motions. The figure is prepared using VMD [65] recurrence properties, in an effort to remove redundant data within the same dynamics region [82]. This method, based on the recurrence properties of the protein dynamics system, can be an added tool in the search for understanding of the relation between dynamics and function for a protein.

\section{Abbreviations}

CRP: Cross recurrence plot; CRQA: Cross recurrence quantification analysis; DET: Determinism; DM: Distance matrix; FNN: False nearest neighbor: MD: Molecular dynamics; MI: Mutual information; NAMD: Not (just) Another Molecular Dynamics program; PCA: Principal component analysis; PDB: Protein data bank; RM: Recurrence matrix; RMSD: Root mean square deviation; RP: Recurrence plot; RQA: Recurrence quantification analysis; RR: Recurrence rate; VMD: Visual molecular dynamics

\section{Acknowledgments}

The author would like to thank the DAAD for supporting his short research visit during the summer of 2016 to the physics department at the University of Regensburg, Germany. He would also like to thank Prof. Dr. Ferdinand Evers, and the physics department family at the University of Regensburg, for hosting him during this visit, and providing a very productive research atmosphere, during which most of this work was accomplished. The author would also like to thank the anonymous reviewers for their helpful comments.

\section{Funding}

Not applicable.

\section{Availability of data and materials}

The datasets used and/or analyzed during the current study are available from the corresponding author on reasonable request.

\section{Author's contributions}

WIK performed all the work in this paper.

Ethics approval and consent to participate

Not applicable. We have no human or animal data involved.

\section{Consent for publication}

Not applicable.

\section{Competing interests}

The author declares that he has no competing interests.

Received: 11 August 2017 Accepted: 15 November 2017 Published online: 28 November 2017

\section{References}

1. Ansari A, Berendsen J, Braunstein D, Cowen BR, Frauenfelder H, Hong MK, Iben IE, Johnson JB, Ormos P, Sauke TB, Scholl R. Rebinding and relaxation in the myoglobin pocket. Biophys Chem. 1987;26(2-3):337-55.

2. Daidone I, Amadei A. Essential dynamics: foundation and applications. Wiley Interdisciplinary Reviews: Computational Molecular Science 2012;2(5):762-770

3. Amadei A, Linssen ABM, Berendsen HJC. Essential dynamics of proteins. Proteins. 1993;17:412-25

4. Garcia AE. Large-amplitude nonlinear motions in proteins. Phys Rev Lett. 1992;68:2696-9.

5. Kitao A, Hirata F, Go N. The effects of solvent on the conformation and the collective motions of protein-normal mode analysis and moleculardynamics simulations of melittin in water and in vacuum. Chem Phys. 1991; 158:447-72.

6. David CC, Jacobs DJ. Principal component analysis: a method for determining the essential dynamics of proteins. Methods Mol Biol. 2014; 1084:193-226.

7. Sittel F, Jain A, Stock G. Principal component analysis of molecular dynamics: on the use of Cartesian vs. internal coordinates. J Chem Phys. 2014:141(014111):1-9.

8. Ichiye T, Karplus M. Collective motions in proteins: a covariance analysis of atomic fluctuations in molecular dynamics and normal mode simulations. Proteins. 1991;11(3):205-17. 
9. Kitao A, Go N. Investigating protein dynamics in collective coordinate space. Curr Opin Struct Biol. 1999;9(2):164-9.

10. Maisuradze GG, Liwo A, Scheraga HA. Principal component analysis for protein folding dynamics. J Mol Biol. 2009;385(1):312-29.

11. Kufareva I, Abagyan I. Methods of protein structure comparison. Methods Mol Biol. 2012:857:231-57.

12. Eckmann JP, Oliffson KS, Ruelle D. Recurrence plots of dynamical systems. Europhys Lett. 1987;4(9):973-7.

13. Marwan N. A historical review of recurrence plots. Eur Phys J-Spec Top. 2008;164(1):3-12.

14. Marwan N, Romano MC, Thiel M, Kurths J. Recurrence plots for the analysis of complex systems. Phys Rep. 2007;438(5):237-329.

15. Webber $\mathrm{CL}$, Zbilut JP. Dynamical assessment of physiological systems and states using recurrence plot strategies. J Appl Physiol. 1994;76(2):965-73.

16. Giuliani A, Manetti C. Hidden peculiarities in the potential energy time series of a tripeptide highlighted by a recurrence plot analysis: a molecular dynamics simulation. Phys Rev E. 1996;53(6):6336-40.

17. Manetti C, Ceruso MA, Giuliani A, Webber CL. JP Zbilut. Recurrence quantification analysis as a tool for characterization of molecular dynamics simulations. Phys Rev E. 1999;59(1):992-8.

18. Giuliani A, Benigni R, Sirabella P, Zbilut JP, Colosimo A. Nonlinear methods in the analysis of protein sequences: a case study in rubredoxins. Biophys J. 2000;78(1):136-49.

19. Zbilut JP, Webber CL, Colosimo A, Giuliani A. The role of hydrophobicity patterns in prion folding as revealed by recurrence quantification analysis of primary structure. Protein Eng. 2000;13(2):99-104.

20. Giuliani A, Sirabella P, Benigni R, Colosimo A. Mapping protein sequence spaces by recurrence quantification analysis: a case study on chimeric structures. Protein Eng. 2000;13(10):671-8.

21. Giuliani A, Colafranceschi M, Webber CL, Zbilut JP. A complexity score derived from principal components analysis of nonlinear order measures. Physica A: Stat Mech Appl. 2001;301(1):567-88.

22. Webber CL, Giuliani A, Zbilut JP, Colosimo A. Elucidating protein secondary structures using alpha-carbon recurrence quantifications. Proteins. 2001; 44(3):292-303.

23. Manetti C, Giuliani A, Ceruso MA, Webber CL, Zbilut JP. Recurrence analysis of hydration effects on nonlinear protein dynamics: multiplicative scaling and additive processes. Phys Lett A. 2001;281(5):317-23.

24. Giuliani A, Benigni R, Zbilut JP, Webber CL, Sirabella P, Colosimo A. Nonlinear signal analysis methods in the elucidation of protein sequencestructure relationships. Chem Rev-Columbus. 2002;102(5):1471-92.

25. Zbilut JP, Sirabella P, Giuliani A, Manetti C, Colosimo A, Webber CL. Review of nonlinear analysis of proteins through recurrence quantification. Cell Biochem Biophys. 2002;36(1):67-87.

26. Giuliani A, Tomasi M. Recurrence quantification analysis reveals interaction partners in paramyxoviridae envelope glycoproteins. Proteins. 2002;46(2):171-6.

27. Giuliani A, Benigni R, Colafranceschi M, Chandrashekar I, Cowsik SM. Large contact surface interactions between proteins detected by time series analysis methods: case study on C-phycocyanins. Proteins. 2003:51(2):299-310.

28. Zbilut JP, Colosimo A, Conti F, Colafranceschi M, Manetti C, Valerio MC, Webber $\mathrm{CL}$, Giuliani A. Protein aggregation/folding: the role of deterministic singularities of sequence Hydrophobicity as determined by nonlinear signal analysis of Acylphosphatase and AB(1-40). Biophys J. 2003:85(6):3544-57.

29. Zbilut JP, Giuliani A, Colosimo A, Mitchell JC, Colafranceschi M, Marwan N, Webber $\mathrm{CL}$, Uversky VN. Charge and hydrophobicity patterning along the sequence predicts the folding mechanism and aggregation of proteins: a computational approach. J Proteome Res. 2004;3(6):1243-53.

30. Porrello A, Soddu S, Zbilut JP, Crescenzi M, Giuliani A. Discrimination of single amino acid mutations of the p53 protein by means of deterministic singularities of recurrence quantification analysis. Proteins. 2004:55(3):743-55

31. Li M, Huang Y, Xu R, Xiao Y. Nonlinear analysis of sequence symmetry of beta-trefoil family proteins. Chaos, Solitons Fractals. 2005;25(2):491-7.

32. Ming-Feng L, Yan-Zhao $H$, Yi X. Nonlinear correlations of protein sequences and symmetries of their structures. Chin Phys Lett. 2005;22(4):1006.

33. Colafranceschi M, Colosimo A, Zbilut JP, Uversky VN, Giuliani A. Structurerelated statistical singularities along protein sequences: a correlation study. $J$ Chem Inf Model. 2005;45(1):183-9.

34. Zbilut JP, Chua GH, Krishnan A, Bossa C, Colafranceschi M, Giuliani A. Entropic criteria for protein folding derived from recurrences: six residues patch as the basic protein word. FEBS Lett. 2006;580(20):4861-4.
35. Grover A, Dugar D, Kundu B. Predicting alternate structure attainment and amyloidogenesis: a nonlinear signal analysis approach. Biochem Biophys Res Commun. 2005;338(3):1410-6.

36. Huang Y, Li M, Xiao Y. Nonlinear analysis of sequence repeats of multidomain proteins. Chaos, Solitons Fractals. 2007;34(3):782-6.

37. Zhou Y, Yu Z, Anh V. Cluster protein structures using recurrence quantification analysis on coordinates of alpha-carbon atoms of proteins. Phys Lett A. 2007;368(3):314-9.

38. Mitra J, Mundra P, Kulkarni BD, Jayaraman VK. Using recurrence quantification analysis descriptors for protein sequence classification with support vector machines. J Biomol Struct Dyn. 2007;25(3):289-97.

39. Karakasidis TE, Fragkou A, Liakopoulos A. System dynamics revealed by recurrence quantification analysis: application to molecular dynamics simulations. Phys Rev E. 2007;76(2):021120.

40. Giuliani A, Krishnan A, Zbilut JP, Tomita M. Proteins as networks: usefulness of graph theory in protein science. Curr Protein Pept Sci. 2008;9(1):28-38.

41. Krishnan A, Giuliani A, Zbilut JP, Tomita M. Implications from a network-based topological analysis of ubiquitin unfolding simulations. PLoS One. 2008;3(5):e2149.

42. Angadi S, Kulkarni A. Nonlinear signal analysis to understand the dynamics of the protein sequences. Eur Phys J-Spec Top. 2008;164(1):141-55.

43. Yang Y, Tantoso E, Li K. Remote protein homology detection using recurrence quantification analysis and amino acid physicochemical properties. J Theor Biol. 2008;252(1):145-54.

44. Karnik S, Prasad A, Diwevedi A, Sundararajan V, Jayaraman V. Identification of Defensins employing recurrence quantification analysis and random Forest classifiers. Pattern Recognition and Machine Intelligence. 2009;5909:152-7.

45. Yang JY, Peng ZL, Yu ZG, Zhang RJ, Anh V, Wang D. Prediction of protein structural classes by recurrence quantification analysis based on chaos game representation. J Theor Biol. 2009;257(4):618-26.

46. Namboodiri S, Verma C, Dhar PK, Giuliani A, Nair AS. Application of recurrence quantification analysis (RQA) in biosequence pattern recognition Adv Comput Commun. 2011;190:284-93.

47. Kulkarni A, Karnik S, Angadi S. Analysis of intrinsically disordered regions in proteins using recurrence quantification analysis. Inter J Bifurcation Chaos. 2011:21(04):1193-202.

48. Han GS, Yu Z-G, Anh V. Predicting the subcellular location of apoptosis proteins based on recurrence quantification analysis and the Hilbert-Huang transform. Chinese Physics B. 2011;20(10):0504.

49. Namboodiri S, Giuliani A, Nair AS, Dhar PK. Looking for a sequence based allostery definition: a statistical journey at different resolution scales. J Theor Biol. 2012;304:211-8.

50. Shao $G$, Yuehui $C$. Predict the tertiary structure of protein with flexible neural tree. Intell Comput Theories App. 2012;7390:324-31.

51. Karain WI, Qaraeen NI. The adaptive nature of protein residue networks. Proteins. 2017:85(5):917-23.

52. Karain WI. THz frequency spectrum of protein-solvent interaction energy using a recurrence plot-based Wiener-Khinchin method. Proteins. 2016; 84(10):1549-57.

53. Karain WI, Qaraeen NI. Weighted protein residue networks based on joint recurrences between residues. BMC bioinformatics. 2015;16(1):173.

54. Fataftah $\mathrm{H}$, Karain Wl. Detecting protein atom correlations using correlation of probability of recurrence. Proteins. 2014;82(9):2180-9.

55. Takens F. Detecting strange attractors in turbulence. In: dynamical systems and turbulence, Warwick 1980. Berlin Heidelberg: Springer; 1981. p. 366-81.

56. Anastasios AT. Reconstructing dynamics from observables: the issue of the delay parameter revisited. Int J Bifurcation Chaos. 2007;17(12):4229-43.

57. Kennel MB, Brown R, Abarbanel HDI. Determining embedding dimension for phase-space reconstruction using a geometrical construction. Phys Rev A. 1992:45(6):3403-11.

58. Grassberger $P$, Schreiber T, Schaffrath $C$. Nonlinear time sequence analysis. Int J Bifurcation Chaos. 1991;1(03):521-47.

59. Marwan N. How to avoid potential pitfalls in recurrence plot based data analysis. Int J Bifurcation Chaos. 2011;21(04):1003-17.

60. Dale R, Warlaumont AS, Richardson DC. Nominal cross recurrence as a generalized lag sequential analysis for behavioral streams. Int J Bifurcation Chaos. 2011;21(04):1153-61.

61. Schinkel S, Marwan N, Dimigen O, Kurths J. Confidence bounds of recurrence-based complexity measures. Phys Lett A. 2009;373:2245-50.

62. Marwan N, Schinkel S, Kurths J. Recurrence plots 25 years later-gaining confidence in dynamical transitions. Europhys Lett. 2013;101(2):20007. 
63. Doran JL, Leskiw BK, Aippersbach S, Jensen SE. Isolation and characterization of a beta-lactamase-inhibitory protein from Streptomyces clavuligerus and cloning and analysis of the corresponding gene. J Bacteriol. 1990;172(9):4909-18.

64. Strynadka NC, Jensen SE, Johns K, Blanchard H, Page M, Matagne A, Frere JM, James MNG. Structural and kinetic characterization of a -lactamaseinhibitor protein. Nature. 1994;368(6472):657-9.

65. Strynadka NC, Jensen SE, Alzari PM, James MN. A potent new mode of $\beta$ lactamase inhibition revealed by the $1.7 \AA$ X-ray crystallographic structure of the TEM-1-BLIP complex. Nat Struct Mol Biol. 1996;3(3):290-7.

66. Humphrey W, Dalke A, Schulten K. VMD - Visual Molecular Dynamics. J Molecular Graphics. 1996;14:33-8.

67. James CP, Braun R, Wang W, Gumbart J, Tajkhorshid E, Villa E, Chipot C, Skeel RD, Kale L, Schulten K. Scalable molecular dynamics with NAMD. J Comput Chem. 2005:26:1781-802.

68. Gretes M, Lim DC, de Castro L, Jensen SE, Kang SG, Lee KJ, Strynadka NC. Insights into positive and negative requirements for protein-protein interactions by crystallographic analysis of the $\beta$-lactamase inhibitory proteins BLIP, BLIP-I, and BLP. J Mol Biol. 2009;389(2):289-305.

69. Darden T, Darrin Y, Pedersen L. Particle mesh Ewald: an N. log (N) method for Ewald sums in large systems. J Chem Phys. 1993;98:10089-92.

70. Marwan N. Cross Recurrence Plot Toolbox for Matlab, Reference Manual, Version 5.15, Release 28.6, 2010, http://tocsy.pik-potsdam.de/crp.php.

71. Glykos NM. Software news and updates carma: a molecular dynamics analysis program. J Comput Chem. 2006;27(14):1765-8.

72. Thorndike RL. Who belongs in the family? Psychometrika. 1953:18(4):267-76

73. Henzler-Wildman K, Kern D. Dynamic personalities of proteins. Nature. 2007; 450(7172):964-72.

74. Khodadadi S, Sokolov AP. Protein dynamics: from rattling in a cage to structural relaxation. Soft Matter. 2015;11(25):4984-98.

75. Skliros A, Zimmermann MT, Chakraborty D, Saraswathi S, Katebi AR, Leelananda SP, Kloczkowski A, Jernigan RL. The importance of slow motions for protein functional loops. Phys Biol. 2012;9(1):014001.

76. Panchenko AR, Madej T. Structural similarity of loops in protein families: toward the understanding of protein evolution. BMC Evol Biol. 2005;5(1):10.

77. Bös C, Lorenzen D, Braun V. Specific in vivo labeling of cell surface-exposed protein loops: reactive Cysteines in the predicted gating loop mark a Ferrichrome binding site and a Ligand-induced conformational change of the Escherichia Coli FhuA protein. J Bacteriol. 1998;180(3):605-13.

78. Li C, Banfield MJ, Dennison C. Engineering copper sites in proteins: loops confer native structures and properties to chimeric cupredoxins. J Am Chem Soc. 2007;129(3):709-18.

79. Smith JW, Tachias K, Madison EL. Protein loop grafting to construct a variant of tissue-type plasminogen activator that binds platelet integrin allbß33. J Biol Chem(1995). 1995;270(51):30486-90

80. Yao P, Dhanik A, Marz N, Propper R, Kou C, Liu G, Van Den Bedem H, Latombe JC, Halperin-Landsberg I, Altman RB. Efficient algorithms to explore conformation spaces of flexible protein loops. IEEE/ACM Trans Comput Biol Bioinform. 2008;5(4):534-45.

81. Petrosino J, Rudgers $\mathrm{G}$, Gilbert $\mathrm{H}$, Palzkill T. Contributions of aspartate 49 and phenylalanine 142 residues of a tight binding inhibitory protein of $\beta$ lactamases. J Biol Chem. 1999;274(4):2394-400.

82. Wolf A, Kirschner KN. Principal component and clustering analysis on molecular dynamics data of the ribosomal L11. 23 S sub-domain. J Mol Model. 2013;19(2):539-49.

\section{Submit your next manuscript to BioMed Central and we will help you at every step:}

- We accept pre-submission inquiries

- Our selector tool helps you to find the most relevant journal

- We provide round the clock customer support

- Convenient online submission

- Thorough peer review

- Inclusion in PubMed and all major indexing services

- Maximum visibility for your research

Submit your manuscript at www.biomedcentral.com/submit
Biomed Central 